\title{
Novitates Velloziacearum florae phanerogamicae Sancti Pauli
}

\author{
RENATO DE MELLO-SILVA ${ }^{1}$
}

(recebido: 5 de dezembro de 2003; aceito: 25 de março de 2004)

\begin{abstract}
Novitates Velloziacearum florae phanerogamicae Sancti Pauli). Vellozia obtecta and V. peripherica, two new species of Velloziaceae from northern São Paulo and adjacent areas of Minas Gerais, are described. Their taxonomic relantionships and the characters supporting them as new species are considered. An account of their leaf and pedicel anatomy is also provided. The synonimization of Barbacenia fragrans and B. tricolor under B. tomentosa is also presented and discussed.
\end{abstract}

Key words - Brazil, Minas Gerais, new species, new synonyms, São Paulo, Velloziaceae

RESUMO - (Novitates Velloziacearum florae phanerogamicae Sancti Pauli). São descritas duas espécies novas de Velloziaceae do norte de São Paulo e áreas adjacentes de Minas Gerais, Vellozia obtecta e V. peripherica. São discutidos suas relações taxonômicas e os caracteres que as sustentam como novas. São fornecidas também descrições anatômicas da lâmina foliar e do pedicelo. A sinonimização de Barbacenia fragrans e B. tricolor sob B. tomentosa é também apresentada e discutida.

Palavras-chave - Brasil, espécies novas, Minas Gerais, São Paulo, sinônimos novos, Velloziaceae

\section{Introdução}

Quando do tratamento das Velloziaceae para a Flora do Estado de São Paulo, duas espécies novas foram delimitadas e são aqui descritas. Vellozia obtecta e $V$. peripherica habitam a região de Pedregulho e Rifaina, no norte do estado, em campos e afloramentos rochosos relacionados com as formações da Serra da Canastra, em Minas Gerais, onde estas espécies também ocorrem. Além da morfologia externa, são apresentados caracteres anatômicos da lâmina foliar, largamente considerados na sistemática de Velloziaceae (e.g. Ayensu 1974, Coetzee 1974, Mello-Silva 1991, 2000, Menezes 1971, 1973, Menezes \& Semir 1991, Menezes et al. 1994, Smith \& Ayensu 1974, 1976) e do pedicelo (Mello-Silva 1997, 2000). É apresentada e discutida, também, a sinonimização de Barbacenia fragrans (cujo parátipo foi coletado em São Paulo) e de $B$. tricolor sob $B$. tomentosa. Esta é a segunda vez que espécies de Velloziaceae são descritas com base em material proveniente de São Paulo, 67 anos após o trabalho de Henrard (1937).

\section{Material e métodos}

Para as descrições anatômicas, as secções transversais da porção mediana das lâminas foliares e dos pedicelos foram feitas a mão livre com lâmina de barbear e coradas com azul de astra e fucsina básica, segundo Roeser (1972). O material no

1. Instituto de Biociências, Universidade de São Paulo, Caixa Postal 11461, 05422-970 São Paulo, SP, Brasil. mellosil@usp.br qual foram baseadas as descrições está indicado entre parênteses.

A análise de agrupamento das populações de Barbacenia foi realizada com base em uma matriz de distância calculada com a utilização de "simple matching coefficient" (SMC) como índice de associação e "unweight pair-group method using arithmetic averages" (UPGMA) como método de agrupamento (Sneath \& Sokal 1973, Crisci \& Armengol 1983). Foi utilizado para análise o programa NTSYS (Rohlf 1993) e apresentado o fenograma de consenso estrito. Os caracteres da matriz (tabela 1) são aqueles usados por Henrard (1937) e Martius \& Zuccarini (1823) na descrição de Barbacenia fragrans, $B$. tomentosa e $B$. tricolor e que permitiriam distinguir as três espécies. Alguns têm sido utilizados por autores posteriores (e.g. Smith \& Ayensu 1976) com este propósito. Esses caracteres também foram analisados nas coleções dos herbários SP e SPF classificadas como uma das três espécies acima (tabela 2). Os caracteres 3 5, 7-9, 11-14, 15-17, 18-20 e 22-24 são decodificação binária de caracteres multiestados, procedimento recomendado para coeficientes de associação (Crisci \& Armengol 1983).

\section{Resultados e Discussão}

Vellozia obtecta Mello-Silva, sp. nov. Tipo: BRASIL: São PAulo: Pedregulho, Estreito, Usina de Estreito (Acampamento de Estreito), encosta à margem da represa, próxima ao antigo aeroporto, $20^{\circ} 09^{\prime} 52^{\prime} \mathrm{S}$ e 47¹6'23"'W, elev. 720 m, 18-III-2003 (fl., fr.), D. Sasaki et al. 197 (holótipo SPF 2 exsicatas; isótipos K, MBM, NY). Figuras 1A-C, 2A-C, 3A, B.

Proxime affinis est V. intermediae, a qua hypanthio tuberculis stellato-truncatis, haud glandulosis, densissime obtecto differt. 
Tabela 1. Caracteres usados na circunscrição e distinção de Barbacenia fragrans, B. tomentosa e B. tricolor segundo Martius \& Zuccarini (1823) e Henrard (1937). 3-5, 7-9, 11-14, 15-17, 18-20 e 22-24 são codificação binária de caracteres multiestados.

Table 1. Characters used by Martius \& Zuccarini (1823) and Henrard (1937) in the circumscription and distinction of Barbacenia fragrans, B. tomentosa and B. tricolor. 3-5, 7-9, 11-14, 15-17, 18-20 e 22-24 are binary coding of multistate characters.

\begin{tabular}{|c|c|c|c|}
\hline Caráter & Estado & Caráter & Estado \\
\hline 1. Indumento da lâmina foliar & $\begin{array}{l}\text { não glanduloso }(0) \\
\text { glanduloso }(1)\end{array}$ & 13. Tépalas alaranjadas & $\begin{array}{l}\text { ausentes (0) } \\
\text { presentes (1) }\end{array}$ \\
\hline 2. Número de flores por ramo & $\begin{array}{l}\text { sempre uma (0) } \\
\text { uma ou mais (1) }\end{array}$ & 14. Tépalas amarelas & $\begin{array}{l}\text { ausentes (0) } \\
\text { presentes (1) }\end{array}$ \\
\hline $\begin{array}{l}\text { 3. Hipanto duas vezes maior } \\
\text { que o ovário }\end{array}$ & $\begin{array}{l}\text { não }(0) \\
\operatorname{sim}(1)\end{array}$ & 15. Corona amarela & $\begin{array}{l}\text { ausente }(0) \\
\text { presente }(1)\end{array}$ \\
\hline 4. Hipanto três vezes maior & não (0) & 16. Corona vermelha & $\begin{array}{l}\text { ausente }(0) \\
\text { presente }(1)\end{array}$ \\
\hline $\begin{array}{l}\text { que o ovário } \\
\text { 5. Hipanto quatro ou mais vezes }\end{array}$ & $\begin{array}{l}\operatorname{sim}(1) \\
\text { não }(0)\end{array}$ & 17. Corona alaranjada & $\begin{array}{l}\text { presente }(1) \\
\text { ausente }(0) \\
\text { presente }(1)\end{array}$ \\
\hline $\begin{array}{l}\text { maior que o ovário } \\
\text { 6. Emergências do hipanto }\end{array}$ & $\begin{array}{l}\operatorname{sim}(1) \\
\text { não glandulosas }(0) \\
\text { glandulosas }(1)\end{array}$ & $\begin{array}{l}\text { 18. Anteras maiores que } \\
\text { os lobos da corona }\end{array}$ & $\begin{array}{l}\text { não }(0) \\
\operatorname{sim}(1)\end{array}$ \\
\hline $\begin{array}{l}\text { 7. Hipanto, na região do ovário, } \\
\text { avermelhado }\end{array}$ & $\begin{array}{l}\text { não }(0) \\
\operatorname{sim}(1)\end{array}$ & $\begin{array}{l}\text { 19. Anteras do mesmo comprimento } \\
\text { que os lobos da corona } \\
\text { 20. Anteras menores que os lobos }\end{array}$ & $\begin{array}{l}\text { não (0) } \\
\operatorname{sim}(1) \\
\text { não (0) }\end{array}$ \\
\hline $\begin{array}{l}\text { 8. Hipanto, na região do ovário, } \\
\text { vermelho }\end{array}$ & $\begin{array}{l}\text { não }(0) \\
\operatorname{sim}(1)\end{array}$ & $\begin{array}{l}\text { da corona } \\
\text { 21. Inserção da antera no lobo }\end{array}$ & $\begin{array}{l}\operatorname{sim}(1) \\
\text { no meio }(0)\end{array}$ \\
\hline 9. Hipanto, na região do ovário, verde & não (0) $\operatorname{sim}(1)$ & da corona & no ápice (1) \\
\hline 10. Cor do tubo do hipanto & $\begin{array}{l}\text { avermelhado (0) } \\
\text { vermelho (1) }\end{array}$ & $\begin{array}{l}\text { 22. Ponto de inserção na antera } \\
\text { no quarto proximal }\end{array}$ & $\begin{array}{l}\text { não }(0) \\
\operatorname{sim}(1)\end{array}$ \\
\hline 11. Tépalas vermelho-purpúreas & $\begin{array}{l}\text { ausentes (0) } \\
\text { presentes (1) }\end{array}$ & $\begin{array}{l}\text { 23. Ponto de inserção na antera } \\
\text { próximo ao meio }\end{array}$ & $\begin{array}{l}\text { não (0) } \\
\operatorname{sim}(1)\end{array}$ \\
\hline 12. Tépalas vermelhas & $\begin{array}{l}\text { ausentes }(0) \\
\text { presentes (1) }\end{array}$ & $\begin{array}{l}\text { 24. Ponto de inserção na antera } \\
\text { próximo à base }\end{array}$ & $\begin{array}{l}\text { não }(0) \\
\operatorname{sim}(1)\end{array}$ \\
\hline
\end{tabular}

Caule 5-80 cm alt., 2-5 cm larg. no ápice. Ramos com 10-16 folhas, espirotrísticas. Bainhas foliares palhete, glabras, encobertas pelas folhas. Lâmina foliar 12-24 cm compr., 5-15 mm larg. na base, linear-triangular, plana, ereta a ereto-patente, depois marcescente e reflexa, in sicco plana, glabra, margens serreadas, ápice atenuado. Inflorescência 1(-3)-flora. Brácteas lanceoladas, ca. $6 \mathrm{~cm}$ compr., ca. $8 \mathrm{~mm}$ larg., glabras. Flores com pedicelo de 7-18 cm compr., 2-3 mm diâm., trígono, ou totalmente glabro ou glabro na metade proximal e com emergências estipitado-estreladas esparsa a densamente distribuídas na metade distal. Hipanto na região do ovário 1-2,5 cm compr., 6-12 mm diâm., obovóide, trígono, verde, densamente coberto de emergências truncado-estreladas; tubo do hipanto ca. $2 \mathrm{~mm}$ compr. Tépalas 6-7 cm compr., 2-3 cm larg., elípticas, unguiculadas, violeta, glabras. Estames ca. 30, unidos em falanges de (4-)5, iguais entre si; filetes 1-1,5 cm compr., inseridos na base das tépalas; anteras 1-2 cm compr., amarelas, látero-introrsas; apêndices estaminais presentes, conspícuos. Estilete 2,5-4 cm compr., pouco ultrapassando os estames; estigma 5-8 mm diâm., amarelo. Cápsula 2,5-3 cm compr., 1-1,5 cm larg., oblonga, trígona, castanha, deiscente por fendas apicais, hipanto persistente.

Anatomia foliar (Romero 1658) - Lâmina dorsiventral. Sulcos $1 / 2$ da espessura da lâmina, papilosos. Epiderme na face adaxial 3-seriada, na abaxial uniseriada. Hipoderme aqüífera unisseriada presente na superfície adaxial, estendendo-se à bainha dos feixes e aos sulcos como parênquima aquífero. Parênquima paliçádico com 3-4 camadas de células e parênquima lacunoso distinto. Feixes fibro-vasculares envolvidos por uma bainha de feixe distinta. 1(-3) grandes elementos de vasos presentes em cada feixe. 2 cordões floemáticos em forma de V separados, abaixo do xilema, por fibras. Fibras pericíclicas estendendo-se adaxialmente até o parênquima aquiífero e abaxialmente até a epiderme. Feixes de fibras de 2(-3) células de espessura presentes na superfície adaxial. 
Tabela 2. Matriz de dados e coleções analisadas. Barbacenia fragrans, B. tomentosa e B. tricolor representam as características destas espécies apresentadas nas descrições originais. Entre parênteses, herbários depositários. Caracteres 1 a 24 listados na tabela $1 ; \mathrm{A}=0$ e 1 .

Table 2. Data matrix and collections. Barbacenia fragrans, B. tomentosa e B. tricolor represent the characteristics of the original descriptions. Within parentheses, the herbaria bearing the collections. Characters 1 to 24 are listed in table 1 ; $\mathrm{A}=0 \mathrm{e} 1$.

$\begin{array}{lllllllllllllll}1 & 1 & 1 & 1 & 1 & 1 & 1 & 1 & 1 & 1 & 2 & 2 & 2 & 2 & 2\end{array}$

$\begin{array}{llllllllllllllllllllllll}1 & 2 & 3 & 4 & 5 & 6 & 7 & 8 & 9 & 0 & 1 & 2 & 3 & 4 & 5 & 6 & 7 & 8 & 9 & 0 & 1 & 2 & 3 & 4\end{array}$

Barbacenia fragrans

Barbacenia tomentosa

Barbacenia tricolor

Badini s.n. - Ouro Preto, MG (OUPR 22463, SPF)

Barros 2699 - Itirapina, SP (SP, SPF)

Brügger 21154 - Lima Duarte, MG (CESJ, SPF)

Caiafa s.n. - Juiz de Fora, MG (CESJ 30507, SPF)

Ceccantini 797 - Analândia, SP (HRCB, SP, SPF, UEC)

Ferreira 575 - Altinópolis, SP (SPF, SPFR)

Forzza 2121 - Baependi, MG (CESJ, SPF)

Giulietti CFCR13726 - São João d'El Rei, MG (K, SPF) A

Hashimoto 7260 - São Tomé das Letras, MG (SPF)

Leoni 4485 - Carangola, MG (GFJP, SPF)

Löfgren CGG1093 - Araraquara, SP (SP)

Löfgren CGG2198 - São João da Boa Vista, SP (SP)

Mello-Silva 213 - Lavras, MG (BHCB, CTES, F, K,

MBM, NY, RB, SP, SPF, UB)

Mello-Silva 1600 - São Tomé das Letras, MG(BHCB, A A $00 \begin{array}{lllllllllllllllllllllll} & 0 & 1 & 0 & 1 & \text { A } & \text { A } & 0 & \text { A } & 0 & 1 & 0 & 0 & 0 & 1 & 0 & 1 & 0 & 0 & 1 & 0 & 0 & 1\end{array}$

$\mathrm{K}, \mathrm{MBM}, \mathrm{RB}, \mathrm{SP}, \mathrm{SPF}, \mathrm{UB}, \mathrm{W})$

Mello-Silva CFCR5708 - São Tomé das Letras, $\mathrm{MG}(\mathrm{SPF})$

Mello-Silva CFCR5793 - São Tomé das Letras, $\mathrm{MG}(\mathrm{SPF})$

Menezes 115 - São Tomé das Letras, MG (SPF, US)

Menezes CFSC11211 - Jaboticatubas, MG (SPF)

Menezes CFSC11260 - Santana do Riacho, MG (SPF)

Pires s.n. - Lima Duarte, MG (CESJ 21542, SPF)

Scatena CFCR11856 - Tiradentes, MG (SPF)

Tameirão Neto 6 - Tiradentes, MG (BHCB, SPF)

Teixeira s.n. - Itabirito, MG (BHCB 24467, SPF)

Rezende s.n. - Itutinga, MG (SPF 151325)

Valente 149 - Mantena, MG (SPF, VIC)

Vasconcelos s.n. - Belo Horizonte, MG (BHCB

36109, SPF)

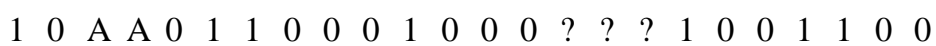

$\begin{array}{llllllllllllllllllllllll}0 & 1 & 0 & 1 & 0 & 0 & 0 & 1 & 0 & 1 & 0 & 1 & 0 & 0 & \text { ? } & \text { ? } & \text { ? } & 1 & 0 & 0 & 1 & 0 & 1 & 0\end{array}$

$\begin{array}{llllllllllllllllllllllll}1 & 1 & 0 & 1 & 0 & 1 & 0 & 1 & 0 & 1 & 0 & 0 & 1 & 0 & 1 & 0 & 0 & 0 & 1 & 0 & 1 & 0 & 0 & 1\end{array}$

$\begin{array}{llllllllllllllllllllllll}0 & 1 & 0 & 0 & 1 & 1 & 0 & 1 & 0 & 1 & 0 & 1 & 0 & 0 & 0 & 1 & 0 & 1 & 0 & 0 & 0 & 0 & 0 & 1\end{array}$

$\begin{array}{llllllllllllllllllllllll}1 & 0 & 0 & 1 & 0 & 1 & 0 & 1 & 0 & 1 & 0 & 1 & 0 & 0 & 0 & 1 & 0 & 1 & 0 & 0 & 0 & 0 & 0 & 1\end{array}$

$\begin{array}{llllllllllllllllllllllll}0 & 1 & 0 & 0 & 1 & 1 & 1 & 0 & 0 & 0 & 1 & 0 & 0 & 0 & 0 & 1 & 0 & 1 & 0 & 0 & 0 & 1 & 0 & 0\end{array}$

A $1 \begin{array}{lllllllllllllllllllllll} & 0 & 0 & 1 & 1 & 1 & 0 & 0 & 0 & 1 & 0 & 0 & 0 & 0 & 1 & 0 & 1 & 0 & 0 & 0 & 1 & 0 & 0\end{array}$

$\begin{array}{llllllllllllllllllllllll}1 & 0 & 0 & 1 & 0 & 1 & 1 & 0 & 0 & 0 & 0 & 0 & 1 & 0 & 0 & 0 & 1 & 1 & 0 & 0 & 0 & 1 & 0 & 0\end{array}$

$\begin{array}{llllllllllllllllllllllll}1 & 1 & 0 & 1 & 0 & 1 & 0 & 1 & 0 & 1 & 0 & 1 & 0 & 0 & 0 & 1 & 0 & 1 & 0 & 0 & 0 & 0 & 0 & 1\end{array}$

$\begin{array}{llllllllllllllllllllllll}1 & 1 & 1 & 0 & 0 & 1 & 0 & 0 & 1 & 0 & 0 & 0 & 0 & 1 & 1 & 0 & 0 & 0 & 1 & 0 & 1 & 0 & 0 & 1\end{array}$

A $0 \begin{array}{lllllllllllllllllllllll} & 0 & 1 & 0 & 1 & 1 & 0 & 0 & 0 & 0 & 1 & 0 & 0 & 0 & 1 & 0 & 1 & 0 & 0 & 1 & 1 & 0 & 0\end{array}$

$\begin{array}{llllllllllllllllllllllll}1 & 1 & 0 & 1 & 0 & 1 & 1 & 0 & 0 & 0 & 0 & 0 & 0 & 1 & 0 & 0 & 1 & 1 & 0 & 0 & 1 & 0 & 0 & 1\end{array}$

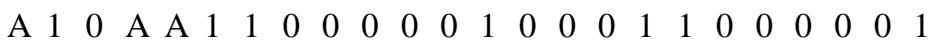

$\begin{array}{llllllllllllllllllllllll}\mathrm{A} & 1 & 0 & 1 & 0 & 1 & 1 & 0 & 0 & 0 & 0 & 1 & 0 & 0 & \text { ? } & \text { ? } & \text { ? } & 1 & 0 & 0 & 1 & 1 & 0 & 0\end{array}$

$\begin{array}{llllllllllllllllllllllll}1 & 1 & 0 & 1 & 0 & 1 & 0 & 1 & 0 & 1 & 1 & 0 & 0 & 0 & \text { ? } & \text { ? } & \text { ? } & 1 & 0 & 0 & 1 & 0 & 0 & 1\end{array}$

$\begin{array}{llllllllllllllllllllllll}1 & 0 & \mathrm{~A} & \mathrm{~A} & 0 & 1 & 1 & 0 & 0 & 0 & 0 & 0 & 1 & 0 & 0 & 1 & 0 & \mathrm{~A} & \mathrm{~A} & 0 & 1 & \mathrm{~A} & 0 & \mathrm{~A}\end{array}$

$\begin{array}{llllllllllllllllllllllll}\mathrm{A} & 0 & 0 & \mathrm{~A} & \mathrm{~A} & 1 & 0 & 1 & 0 & 1 & 0 & 1 & 0 & 0 & 0 & 1 & 0 & 1 & 0 & 0 & 1 & 0 & 0 & 1\end{array}$

$\begin{array}{llllllllllllllllllllllll}0 & 1 & 0 & 1 & 0 & \text { A } & 1 & 0 & 0 & 0 & 0 & 1 & 0 & 0 & 0 & 1 & 0 & 0 & 0 & 1 & 1 & 1 & 0 & 0\end{array}$

$\begin{array}{llllllllllllllllllllllll}0 & 1 & 0 & 1 & 0 & 1 & 1 & 0 & 0 & 0 & 0 & 0 & 1 & 0 & 0 & 0 & 1 & 1 & 0 & 0 & 1 & 1 & 0 & 0\end{array}$

$\begin{array}{llllllllllllllllllllllll}1 & 1 & 0 & 0 & 1 & 1 & 1 & 0 & 0 & 0 & 0 & 0 & 1 & 0 & 0 & 0 & 1 & 1 & 0 & 0 & 1 & 0 & 0 & 1\end{array}$

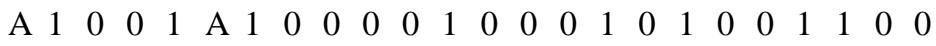

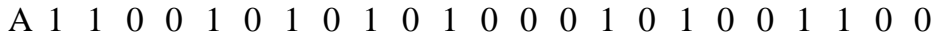

$\begin{array}{llllllllllllllllllllllll}1 & 0 & 0 & 1 & 0 & 1 & 1 & 0 & 0 & 0 & 0 & 0 & 1 & 0 & 0 & 0 & 1 & 1 & 0 & 0 & 1 & 1 & 0 & 0\end{array}$

$\begin{array}{llllllllllllllllllllllll}1 & 0 & 0 & 1 & 0 & 1 & 0 & 1 & 0 & 1 & 0 & 1 & 0 & 0 & 0 & 1 & 0 & 0 & 1 & 0 & 1 & 0 & 0 & 1\end{array}$

$\begin{array}{llllllllllllllllllllllll}1 & 1 & 0 & 1 & 0 & 1 & 1 & 0 & 0 & 0 & 0 & 1 & 0 & 0 & 0 & 1 & 0 & 1 & 0 & 0 & 1 & 0 & 0 & 1\end{array}$

$\begin{array}{llllllllllllllllllllllll}1 & 1 & 0 & \text { A } & \text { A } & 1 & 0 & 1 & 0 & 1 & 0 & 1 & 0 & 0 & 0 & 1 & 0 & 0 & 1 & 0 & 1 & 0 & 0 & 1\end{array}$

$\begin{array}{llllllllllllllllllllllll}1 & 0 & 0 & 1 & 0 & 1 & 1 & 0 & 0 & 0 & 0 & 0 & 1 & 0 & 0 & 0 & 1 & 1 & 0 & 0 & 0 & 0 & 0 & 1\end{array}$

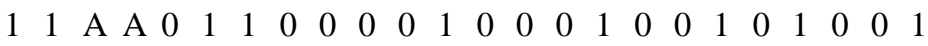

Anatomia do pedicelo (Romero 1658) - Triangular em seção transversal. 12 feixes fibro-vasculares. Cinturão de células esclerificadas presente.

Parátipos: BRASIL: Minas Gerais: São Roque de Minas, Parque Nacional da Serra da Canastra, morro próximo à sede administrativa, 10-I-1995 (fl.), R. Romero et al. 1658 (HUFU, SPF); Sacramento, Parque Nacional da Serra da Canastra, Guarita de Sacramento, 14-X-1994 (fl.), R. Romero et al. 1253
(HUFU, SPF); id., ib., 24-IX-1995 (fl., fr.), R. Romero et al. 2728 (HUFU, SPF); id., ib., 18-XI-1995 (fl.), R. Romero et al. 3035 (HUFU, SPF). São PaUlo: Pedregulho, Estreito, Fazenda 3 Irmãos ("3 Irmãs"), 16-I-1997 (fl.), A.D. Faria et al. 97/149 (SPF, UEC).

Vellozia obtecta é semelhante a $V$. intermedia Seub., com a qual compartilha as folhas marcescentes e reflexas sobre o caule, a presença de apêndices estaminais e o tipo de padrão anatômico foliar, com 


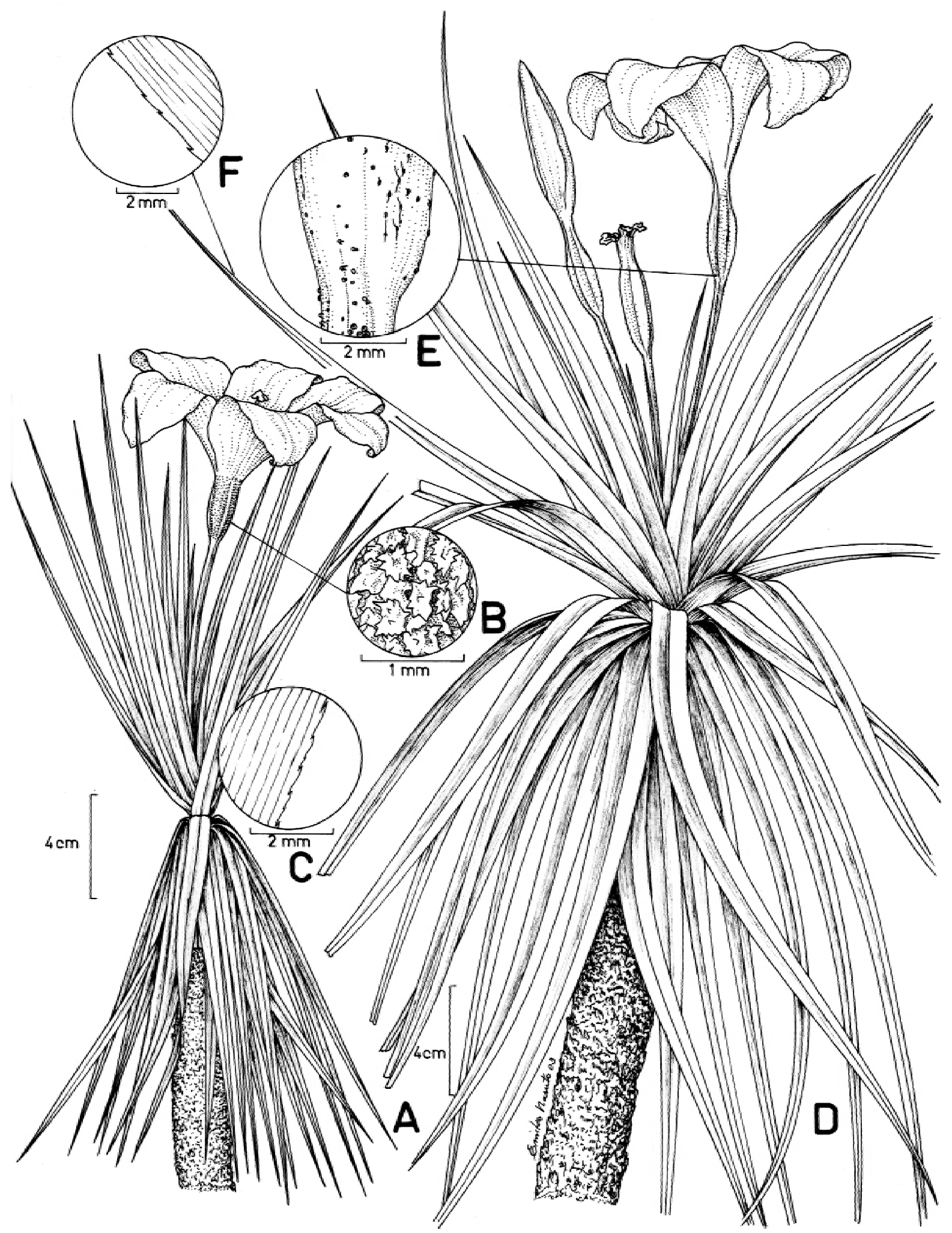

Figura 1. A-C. Vellozia obtecta. A. Hábito com flor. B. Detalhe das emergências do hipanto. C. Detalhe da margem da lâmina foliar. D-F. Vellozia peripherica. D. Hábito com flor e fruto. E. Detalhe das emergências na base do hipanto. F. Detalhe da margem da lâmina foliar. (A-C: Romero 3035, D-F: Mello-Silva et al. 1951).

Figure 1. A-C. Vellozia obtecta. A. Habit with flower. B. Detail of hypanthial emergences. C. Detail of leaf margin. D-F. Vellozia peripherica. D. Habit with flower and fruit. E. Detail of the emergences from the base of the hypanthium. F. Detail of leaf margin. (A-C: Romero 3035, D-F: Mello-Silva et al. 1951). 
parênquima aqüífero sobre feixes e sulcos. Diferem pelo tipo de emergências que recobrem o hipanto. Em $V$. intermedia as emergências são ventosiformes, glandulares e esparsamente distribuídas; em V. obtecta, as emergências são estrelado-truncadas, não glandulares e densamente distribuídas. As duas espécies são disjuntas. Vellozia obtecta ocorre em Pedregulho (São Paulo) e na Serra da Canastra e Patrocínio (Minas Gerais) (figura 4); Vellozia intermedia é restrita à porção central da Cadeia do Espinhaço de Minas Gerais, ocorrendo no Planalto de Diamantina e em disjunções a leste (Rio Vermelho) e a oeste (Serra do Cabral).

Vellozia peripherica Mello-Silva, sp. nov. Tipo: BRASIL: São Paulo: Pedregulho, Igaçaba, Morro
Solteiro, 12-XI-1994 (fl.), W. Marcondes Ferreira et al. 1010 (holótipo SP; isótipos ESA, HRCB, SPF, SPFR, UEC).

Figuras 1D-F, 2D-F, 3C, D.

Velloziae sincoranae proxime est affinis et tubo hypanthii cc. 1/3 longitudine ovarii, pedicello apicem versus plerumque sparsim stipitatoglanduloso, tepalis albis vel violaceis atque planta generatim minus robusta ab ea differt.

Caule simples ou pouco ramificado, 0,4-1 m alt., 2-5 cm larg. no ápice. Ramos com 8-15 folhas, espirotrísticas. Bainhas foliares palhete, glabras, resinosas, encobertas pelas folhas. Lâmina foliar 18-39 cm compr., 7-20 mm larg. na base, linear-

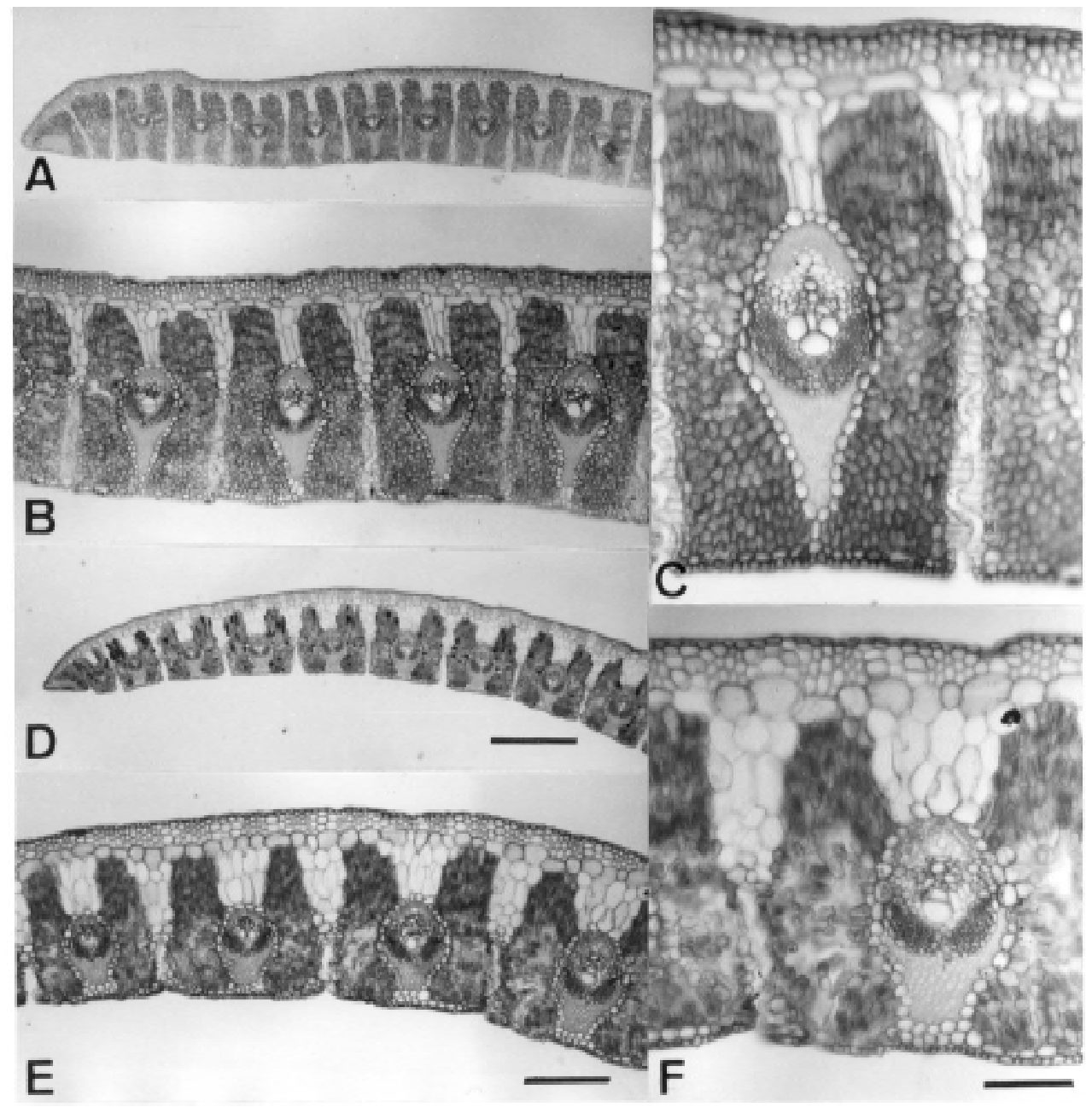

Figura 2. Corte transversal da região mediana da folha. A-C. Vellozia obtecta. D-F. V. peripherica. Barras: A =0,5 mm, B =0,25 mm, $\mathrm{C}=0,12 \mathrm{~mm}$. A e D, B e F, C e F estão na mesma ampliação, respectivamente. (A-C: Romero 1658, D-F: Farinaccio et al. 240).

Figure 2. Cross section of leaf blade. A-C. Vellozia obtecta. D-F. V. peripherica. Bars: A =0,5 mm, B =0,25 mm, C =0,12 mm. A e D, B e F, C e F are in the same magnification, respectively. (A-C: Romero 1658, D-F: Farinaccio et al. 240). 
triangular, arcuada, ereta a ereto-patente, depois marcescente e reflexa, in sicco involuta na metade proximal e revoluta na distal, glabra, margens inteiras na metade proximal, serreadas na distal, ápice atenuado. Inflorescência 1-5-flora. Brácteas oval-lanceoladas, 5-9 cm compr., 5-14 mm larg., resinosas, glabras. Flores odoríferas. Pedicelo 9-23 cm compr., ca. 2 mm diâm., trígono, verde-amarelado, mais claro na base, glabro na metade proximal e com curtas emergências estipitado- glandulares esparsamente distribuídas na metade distal, raramente totalmente glabro, resinoso. Hipanto na região do ovário 2-3,5 cm compr., 6-15 mm larg., elipsóide a ovóide, trígono, verde-amarelado a verde-acastanhado, resinoso, ou glabro ou com poucas emergências semelhantes às do pedicelo sobre os ângulos ou sobre os ângulos e na região proximal ou, raramente, por todo o hipanto; tubo do hipanto 8-20 mm compr., 5-10 mm diâm., lanceolóide, trígono, verde-amarelado a verde-
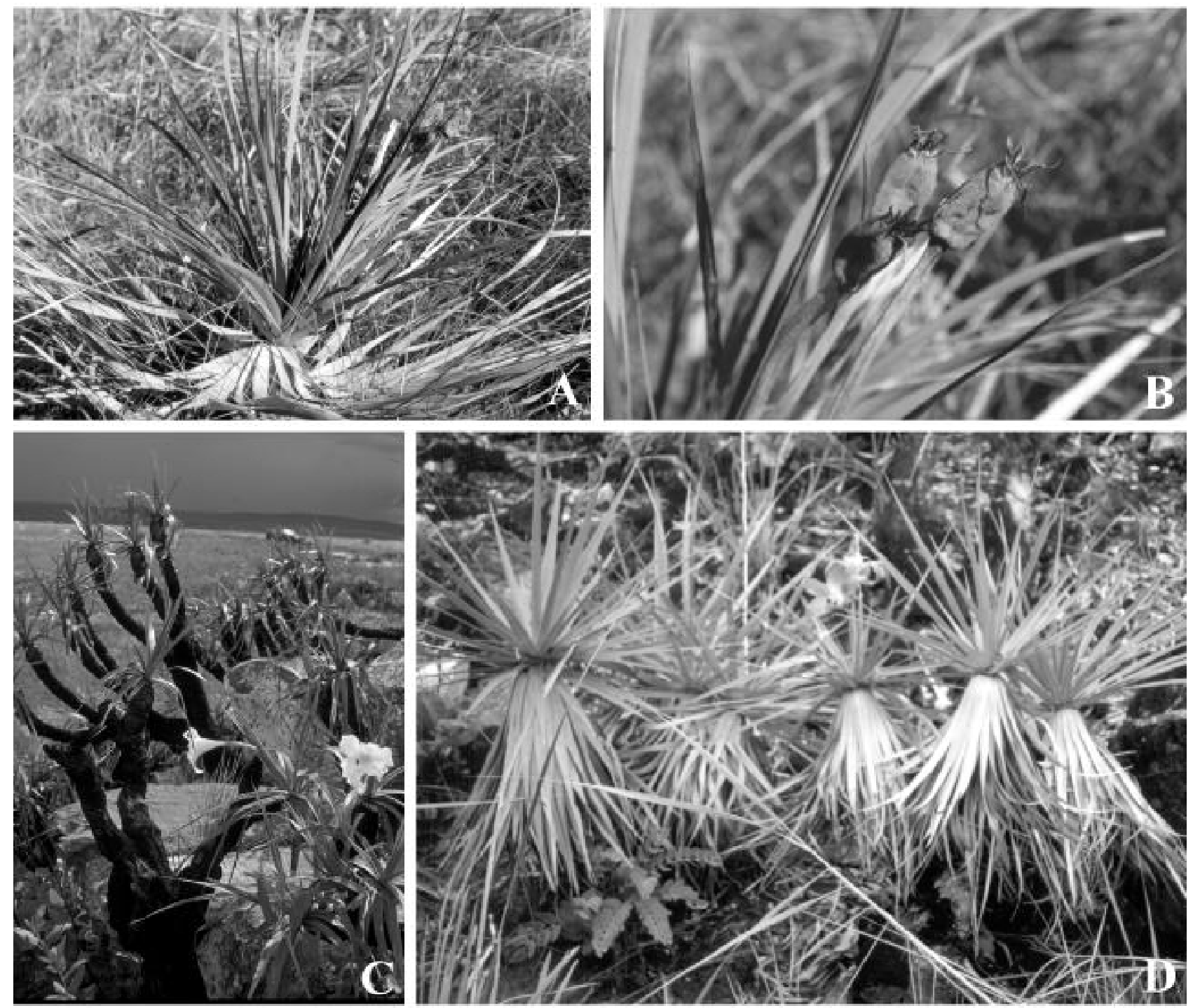

Figura 3. A-B. Vellozia obtecta, hábito com frutos, em Estreito, Pedregulho. C-D. V. peripherica. C. Hábito com bainhas foliares carbonizadas por queimada e flores, na Serra da Canastra, Minas Gerais. D. Hábito ctm flores, no Parque Estadual das Furnas de Bom Jesus, Pedregulho. (A, B: Sasaki et al. 197, C: Farinaccio et al. 240, D: Mello-Silva et al. 1949). Fotos: R. Mello-Silva (A, B), M. A. Farinaccio (C), D. Sasaki (D).

Figure 3. A-B. Vellozia obtecta, habit with fruits, at Estreito, Pedregulho. C-D. V. peripherica. C. Habit with burned leaf sheaths and flowers, at Serra da Canastra, Minas Gerais. D. Habit with flowers, at Furnas de Bom Jesus State Park, Pedregulho. (A, B: Sasaki et al. 197, C: Farinaccio et al. 240, D: Mello-Silva et al. 1949). Fotos: R. Mello-Silva (A, B), M. A. Farinaccio (C), D. Sasaki (D). 


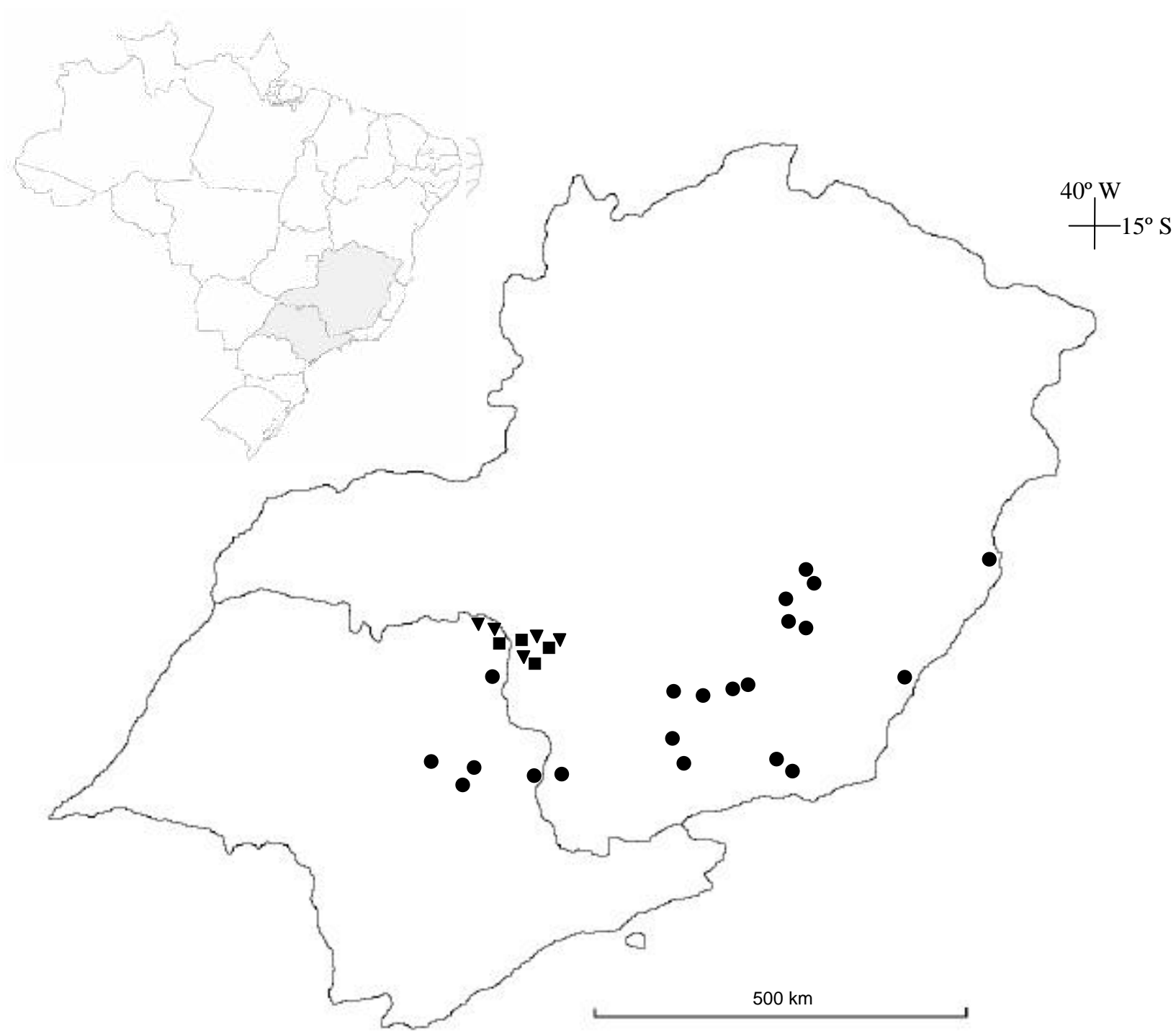

Figura 4. Distribuição geográfica de Barbacenia tomentosa $(\bullet)$, Vellozia obtecta $(\bullet)$ e V. peripherica $(\boldsymbol{\nabla})$.

Figure 4. Geographical distribution of Barbacenia tomentosa $(\bullet)$, Vellozia obtecta $(\bullet)$ e V. peripherica $(\boldsymbol{\nabla})$.

acastanhado, glabro, persistente na frutificação. Tépalas 5-9,5 cm compr., 2-4 cm larg., oblongo-elípticas a elípticas, ou totalmente brancas ou brancas na metade proximal e violeta na distal ou totalmente violeta, glabras. Estames (28-)36, em falanges de seis, iguais entre si; filetes ca. 1,5-2 cm compr., inseridos no ápice do hipanto, brancos; anteras 1,5-2,5 cm compr., amarelas, latrorsas; apêndices estaminais presentes, pouco conspícuos. Estilete 5,5-7,5 cm compr., branco, ultrapassando os estames; estigma 6-10 mm diâm., amarelo. Cápsula 4-4,5 cm compr., 1-1,5 cm larg., oblongo-elipsóide a oblonga, trígona, castanho-clara, deiscente por fendas apicais, hipanto persistente.

Anatomia foliar (Farinaccio et al. 240) - Lâmina dorsiventral. Sulcos 1/2 a 1/3 da espessura da lâmina, papilosos. Epiderme na face adaxial 3-4-seriada, na abaxial unisseriada. Hipoderme aqüífera bisseriada presente na superfície adaxial, estendendo-se à bainha dos feixes e aos sulcos como parênquima aqüífero. Parênquima paliçádico com 3-4 camadas de células e parênquima lacunoso distinto. Feixes fibro-vasculares envolvidos por uma bainha de feixe distinta. 1(-2) grandes elementos de vasos presentes em cada feixe. 2 cordões floemáticos em forma de $\mathrm{V}$ separados, abaixo do xilema, por fibras. Fibras pericíclicas estendendo-se adaxialmente até o parênquima aquíffero e abaxialmente até a epiderme. Feixes de fibras de 2-3 células de espessura presentes na superfície adaxial. 
Anatomia do pedicelo (Farinaccio et al. 240) Triangular em seção transversal. 24-36 feixes fibrovasculares. Cinturão de células esclerificadas ausente.

Parátipos: BRASIL: Minas GeRAIS: Delfinópolis, Serra da Gurita, próximo à estrada Delfinópolis Sacramento, elev. 900 m, 8-I-1996 (fl.), V.C. Souza et al. 9844 (ESA, SPF); id., 21-XII-1999 (fl.), A.C.B. Silva 127 (SPF, SPFR); São Roque de Minas, arredores do Parque Nacional da Serra da Canastra, 20 $155^{\prime} 62^{\prime \prime}$ -

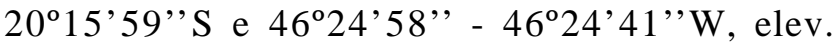
1.095-1.176 m, 12-I-1994 (fl), A.M. Giulietti et al. CFCR13591 (BHCB, K, MBM, SPF); Parque Nacional da Serra da Canastra, topo da cachoeira Casca d'Anta, 20-XII-1991 (fl.), F. Rivadávia Lopes 38 (SPF); id., ib., 20-IV-1994 (fl.), R. Romero et al. 954 (HUFU, SPF); id., morro próximo à sede administrativa, 7-XII1994 (fl., fr.), J.N. Nakajima \& R. Romero 645 (HUFU, SPF); id., $4 \mathrm{~km}$ após entrada para a cachoeira Casca d'Anta, 9-XII-1994 (fl.), J.N. Nakajima \& R. Romero 704 (HUFU, SPF); id., ib., 9-XII-1994 (fl., fr.), J.N. Nakajima \& R. Romero 711 (HUFU, SPF); id., estrada para Sacramento, após entrada da cachoeira, 18-III-1995 (fr.), J.N. Nakajima et al. 885 (HUFU, SPF); id., Cachoeira dos Rolinhos, 22-XI-1998 (fl.), M.A. Farinaccio et al. 204 (NY, RB, SPF); id., Curral de Pedras, 15-XII-1998 (fl.), M.A. Farinaccio \& E.M. Campos Filho 240 (SPF, US); id., estrada São Roque de Minas - Sacramento, a $2 \mathrm{~km}$ da portaria de São Roque, 9-XI-2002 (fl.), R. Mello-Silva et al. 1989 (BHCB, CTES, F, G, HUEFS, K, NY, SP, SPF, US). SÃo PAUlo: Pedregulho, Estreito, Fazenda 3 Irmãos, 18-I-1996 (fl.), W. Marcondes Ferreira \& R. Belinello 1261 (SP, SPF, UEC); id., ib., 5-XI-1997 (fl.), W. Marcondes Ferreira et al. 1452 (HRCB, SPF, SP, UEC); id., ib., 26-IX-2002 (fl., fr.), R. Mello-Silva et al. 1951 (SPF); Parque Estadual das Furnas do Bom Jesus, escarpas a oeste da confluência dos rios Bom Jesus e Pedregulho, 25-XI-2002 (fr.), R. Mello-Silva et al. 1949 (SPF).

Vellozia peripherica pertence ao grupo ( $V$. alata ((V. punctulata (V. caput-ardeae, V. hatschbachii)) (V. compacta (V. epidendroides, V. minima)))), definido (Mello-Silva 2000) pelas arqueomorfias: a) filotaxia espirotrística, b) apêndices estaminais presentes e c) cápsula poricida, e pelas sinapomorfias: a) tubo do hipanto mais curto que o ovário e b) parênquima aquífero estendendo-se adaxialmente aos feixes e aos sulcos. $V$. peripherica compartilha com V. alata L.B. Sm., da Serra do Cipó, Minas Gerais (Sazima 1978, N.L. Menezes dados não publicados) e com $V$. sincorana L.B. Sm. \& Ayensu, da Chapada Diamantina, Bahia
(A. Conceição, dados não publicados) a capacidade de florescer rápida e abundantemente após queimadas (figura 3C). Vellozia peripherica habita área periférica à distribuição do grupo, com populações em Pedregulho e Rifaina (São Paulo), e Delfinópolis e Serra da Canastra (Minas Gerais) (figura 4). As outras espécies do grupo ocorrem na Cadeia do Espinhaço, da Serra do Cipó, centro de Minas Gerais, a Lençóis, na Chapada Diamantina, Bahia. A espécie mais semelhante a $V$. peripherica é $V$. sincorana que, embora também não analisada por Mello-Silva (2000), apresenta características que permitem inseri-la no grupo acima.

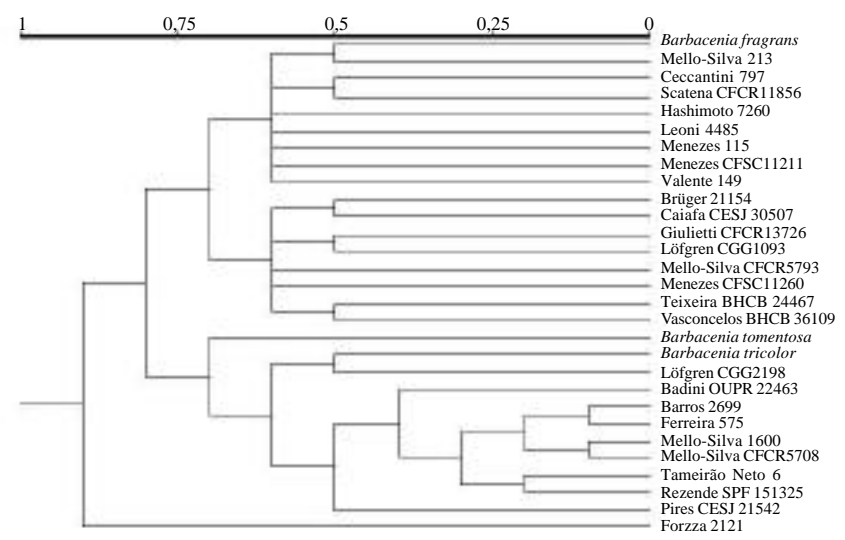

Figura 5. Fenograma de consenso de coleções de Barbacenia tomentosa.

Figure 5. Consensus phenogram of the collections of Barbacenia tomentosa.

Vellozia peripherica é polimórfica quanto à cor das tépalas. Este fenômeno é comum em algumas espécies de Vellozia, onde a cor pode variar de violeta a branco, ou vice-versa, numa mesma população (vide Ayensu 1973, Mello-Silva 2000). Em V. peripherica, porém, a variação parece estar associada a um gradiente geográfico. As populações de São Paulo têm tépalas violeta. Na região de Delfinópolis, Minas Gerais, os indivíduos apresentam tépalas ou violeta ou brancas com ápice violeta. Esta última situação, tépalas brancas com ápice violeta, é pouco comum mais ao norte, na Serra da Canastra, Minas Gerais, onde a maioria dos indivíduos exibe tépalas brancas.

Barbacenia tomentosa Mart. in Mart. \& Zucc., Nov. Gen. sp. pl. 1:18, tab. 11. 1823. Tipo: BRASIL: MINAS GERAIS: C.P.F. Martius s.n. (holótipo M?, n.v.).

= Barbacenia tricolor Mart. in Mart. \& Zucc., Nov. Gen. sp. pl. 1:18, tab. 10. 1823. Tipo: BRASIL: 
Minas Gerais: C.F.P. Martius s.n. (holotypus M?,n.v.). Syn. nov.

= Barbacenia fragrans Goethart \& Henrard in Henrard, Blumea 2:345. 1937. Tipo: BRASIL: MinAS Gerais: Caldas, 1-II-1876 (fl.), C.W.H. Mosén 4444 (holótipo S, n.v., foto K, SPF). Syn. nov.

Barbacenia tomentosa e B. tricolor foram descritas por Martius na mesma obra (Martius \& Zuccarini 1823). Mais tarde, Goethart \& Henrard in Henrard (1937) publicaram B. fragrans. B. tricolor ocorreria em Minas Gerais, assim como B. tomentosa, que ocorreria mais precisamente entre São João d'El Rei e Ouro Preto ("Vila Rica") e também no Planalto de Diamantina ("distrito diamantino") (Martius \& Zuccarini 1823). B. fragrans ocorreria no sudoeste de Minas Gerais e centro-norte de São Paulo e seu nome é uma alusão ao odor de Pelargonium que emanaria das glândulas do perianto (Henrard 1937).

Os estados dos caracteres que distinguiriam as três espécies, segundo seus respectivos autores, estão listados na tabela 1 e plotados na matriz da tabela 2. Uma análise da matriz da tabela 2 por UPGMA resultou em seis fenogramas. O fenograma de consenso estrito (figura 5) pode ser dividido em três grandes grupos. No primeiro, está uma coleção de Baependi (Forzza 2121). No segundo situa-se o tipo (descrição) de Barbacenia fragrans juntamente com coleções oriundas de uma grande amplitude geográfica. $\mathrm{O}$ mesmo se dá no terceiro agrupamento, onde estão os tipos (descrições) de B. tomentosa e B. tricolor. Estes três grandes agrupamentos não correspondem às três espécies estabelecidas. Barbacenia tomentosa e B. tricolor encontram-se no mesmo agrupamento e $B$. tricolor tem Löfgren CGG2198 (parátipo de B. fragrans) como coleção mais semelhante. As diversas coleções de São Tomé das Letras apresentam variações que as situam em vários agrupamentos distintos. O mesmo sucede com as coleções de Lima Duarte e as de Tiradentes/São João d'El Rei. Em alguns casos, uma única coleção pode apresentar espécimes com características que permitiriam incluí-los em cada uma das três espécies. Por exemplo, na coleção de Lavras (Mello-Silva 213) há alguns espécimes com anteras maiores que os lobos da corona e outros com anteras menores que os lobos da corona. Isto permitiria classificá-los como B. fragrans/B. tomentosa e como B. tricolor, respectivamente. Do mesmo modo, alguns espécimes apresentam anteras com ponto de inserção próximo à base e outros, anteras com ponto de inserção no quarto proximal. Isto permitiria classificá-los ou como B. tricolor ou como B. fragrans, respectivamente. Os estados dos caracteres 3-5 nesta coleção permitiriam classificar alguns espécimes ou como $B$. fragrans ou como B. tomentosa/B. tricolor, respectivamente. Assim, a identidade das três espécies não se sustenta $\mathrm{e}$ elas são aqui sinonimizadas.

O odor de Pelargonium, característico de B. fragrans, segundo Mosén (fide Henrard 1937), não foi anotado nem observado nos materiais analisados.

Agradecimentos - Ilustrações e fotografias foram em parte financiadas pela Fapesp. Agradeço a Emiko Naruto pelos desenhos, a Marina Millanelo do Amaral pela assistência no Laboratório de Anatomia Vegetal, a Déborah Y.A. Cursino dos Santos pela assistência na análise fenética, aos dois assessores anônimos pelas sugestões, a Denise Sasaki e Maria Ana Farinaccio pelas fotos de Vellozia peripherica e a Amauri Cesar Marcato pela impressão das fotos dos hábitos; aos organizadores da Flora do Estado de São Paulo, Maria das Graças Lapa Wanderley, George J. Shepherd e Ana Maria Giulietti, e aos organizadores da Flora do Parque Nacional da Serra da Canastra, Rosana Romero e Jimy N. Nakajima, pelo convite à elaboração dessas Floras, impulso inicial deste trabalho. Agradeço ao CNPq pela bolsa de produtividade em pesquisa, processo 300308/96-5.

\section{Referências bibliográficas}

AYENSU, E.S. 1973. Biological and morphological aspects of the Velloziaceae. Biotropica 5:135-149.

AYENSU, E.S. 1974. Leaf anatomy and systematics of New World Velloziaceae. Smithsonian Contributions to Botany 15:1-125.

COETZEE, H. 1974. Anatomy of the leaves of the Velloziaceae in South Africa and South West Africa and a key based on leaf anatomy. Dinteria 10:19-33.

CRISCI, J.V. \& ARMENGOL, M.F.L. 1983. Introduccion a la teoria y practica de la taxonomia numerica. Secretaria General de la Organización de los Estados Americanos, Washington.

HENRARD, J.T. 1937. Velloziaceae americanae nonnulae novae vel minus cognitae. Blumea 2:339-384.

MARTIUS, C.F.P. \& ZUCCARINI, J.G. 1823. Nova Genera et species plantarum. Typis Lindaueri, München, v.1.

MELLO-SILVA, R. 1991. A new species of Vellozia from the Espinhaço Range, Brazil, with some consideration on the section Xerophytoides. Kew Bulletin 46:321-326.

MELLO-SILVA, R. 1997. Vellozia sessilis L.B.Sm. ex MelloSilva (Velloziaceae), espécie nova de Goiás, Brasil. Boletim de Botânica da Universidade de São Paulo 16:65-69.

MELLO-SILVA, R. 2000. Partial cladistic analysis of Vellozia and characters for the phylogeny of Velloziceae. In Monocots: systematics and evolution (K.L. Wilson \& D.A. Morrison, eds.). CSIRO, Melbourne, p.505-522. 
MENEZES, N.L. 1971. Traqueídes de transfusão no gênero Vellozia Vand. Ciência \& Cultura 23:389-409.

MENEZES, N.L. 1973. Natureza dos apêndices petalóides em Barbacenioideae (Velloziaceae). Boletim de Zoologia e Biologia Marinha, n.s. 30:713-755.

MENEZES, N.L. \& SEMIR, J. 1991. Burlemarxia, a new genus of Velloziaceae. Taxon 40: 413-426.

MENEZES, N.L., MELLO-SILVA, R. \& MAYO, S.J. 1994. A cladistic analysis of the Velloziaceae. Kew Bulletin 49:71-92.

ROESER, K.-R. 1972.Die Nadel der Schwarzkiefer Massenprodukt und Kunstwerk der Natur. Mikrokosmos 61:33-36.
ROHLF, F.J. 1993. Numerical taxonomy and multivariate analysis system, version 1.8. Applied Biostatistics, Setauket.

SAZIMA, M. 1978. Biologia floral de espécies de Velloziaceae na Serra do Cipó, Minas Gerais. Tese de doutorado, Universidade de São Paulo, São Paulo.

SMITH, L.B. \& AYENSU, E.S. 1974. Classification of Old World Velloziaceae. Kew Bulletin 29:181-205.

SMITH, L.B. \& AYENSU, E.S. 1976. A revision of American Velloziaceae. Smithsonian Contributions to Botany 30: i-viii + 1-172.

SNEATH, P.H.A. \& SOKAL, R.R. 1973. Numerical taxonomy. The principles and practice of numerical classification. Freeman, San Francisco. 
